\title{
Revista de alto impacto especializada en Marketing
}

\author{
High impact magazine specialized in Marketing
}

Revista de alto impacto especializada em Marketing

Recibido: enero 2021

Arbitrado: febrero 2021

Aceptado: marzo 2021

Publicado: mayo 2021

\section{Laura Raquel Cardozo Rejas}

lauracardozorejas@gmail.com

https://orcid.org/0000-0003-2496-9847

Universidad Privada de Valle, Santa Cruz-Bolivia
RESUMEN

El objetivo de este artículo fue analizar la difusión de conocimiento desde revistas científicas de alto impacto especializadas en Marketing. El estudio se desarrolló con corte transversal considerando indicadores de impacto de las revistas contenidas en la subcategoría "marketing" de la categoría "negocios, administración y contabilidad" de SCImago Journal \& Country Rank al 2020. Como resultados se obtuvieron: el $86.6 \%$ de las revistas se concentran en Reino Unido y Estados Unidos; $13.4 \%$ de las revistas son de acceso abierto; $1.7 \%$ están indizadas en SciELO y $71.5 \%$ en WoS; el 17\% de las revistas Q1 es de acceso abierto. Se concluyó que las revistas cuya participación es estratégica para la difusión de conocimiento en países en desarrollo son: Journal of Innovation and Knowledge, European Research on Management and Business Economics, Transformations in Business and Economics, Cogent Business and Management, Innovar, Estudios Gerenciales y RAE Revista de Administracao de Empresas.

Palabras clave: Índice de impacto; marketing; difusión de conocimiento; producción científica, revista científica
The objective of this article was to analyze the dissemination of knowledge from high impact scientific journals specialized in Marketing. The study was developed with a cross section considering impact indicators of the journals contained in the subcategory "marketing" of the category "business, administration and accounting" of SCImago Journal \& Country Rank to 2020 . As results were obtained: $86.6 \%$ of the journals are concentrated in the United Kingdom and the United States, $13.4 \%$ of the journals are open access; $1.7 \%$ are indexed in SciELO and $71.5 \%$ in WoS; $17 \%$ of Q1 journals are open access. It was concluded that the journals whose participation is strategic for the dissemination of knowledge in developing countries are: Journal of Innovation and Knowledge, European Research on Management and Business Economics, Transformations in Business and Economics, Cogent Business and Management, Innovar, Studios Geranial's and RAE Business Administration Magazine.

Palabras clave: Impact index; marketing; knowledge dissemination; scientific production, scientific journal
RESUMO

O objetivo deste artigo foi analisar a disseminação do conhecimento de revistas científicas de alto impacto especializadas em Marketing. O estudo foi desenvolvido com um corte transversal considerando indicadores de impacto dos periódicos contidos na subcategoria "marketing" da categoria "negócios, administração e contabilidade" do SCImago Journal \& Country Rank to 2020. Como resultados foram obtidos: $86,6 \%$ dos periódicos estão concentrados no Reino Unido e nos Estados Unidos, $13,4 \%$ dos periódicos são de acesso aberto; $1,7 \%$ são indexados no SciELO e $71,5 \%$ no WoS; $17 \%$ dos periódicos do primeiro trimestre são de acesso aberto. Concluiu-se que os periódicos cuja participação é estratégica para a disseminação do conhecimento em países em desenvolvimento são: Journal of Innovation and Knowledge, European Research on Management and Business Economics, Transformations in Business and Economics, Cogent Business and Management, Innovar, Estudios Gerenciales e Revista RAE Business Administration.

Palabras clave: Índice de impacto; marketing; disseminação de conhecimento; produção científica, revista científica 


\section{INTRODUCCIÓN}

Spinak (1998), postula que la ciencia puede ser entendida como un sistema de producción de información, mayormente registrada y publicada en formatos permanentes. Este registro permite discutir y compartir el conocimiento. En palabras de Espinosa (2019), la investigación reclama su difusión, exponer sus logros, compartir sus resultados [...] pretende su propagación por escrito. (p. 59). Si bien existen otros medios que favorecen la difusión del conocimiento, la comunicación escrita puede ser sometida a filtros que garantizan su veracidad, para posterior difusión y divulgación mediante revistas científicas, congresos, docencia, redes sociales y académicas.

En el ámbito académico, las revistas científicas son consideradas un medio fiable de difusión. Las investigaciones que se publican en estas revistas han sido sometidas a una serie de filtros y revisiones que acreditan su calidad y veracidad (Hanwell et al. 2012). La comunidad científica acude a estas revistas por dos motivos: primero, para conocer el estado del arte del tema que están estudiando; posteriormente, buscando someter sus aportes a revisión y validación. Entonces, haciendo una analogía entre ciencia y empresa, los científicos juegan el papel de consumidores y proveedores.

Según Spinak (1998), bajo la misma analogía, la ciencia tiene dos categorías: insumos y resultados, la medición de ambas es la base de los indicadores científicos. Estos indicadores buscan medir los resultados (impacto) de los insumos (producción científica). El impacto tradicionalmente se enfocaba en cuantificar la difusión, actualmente también mide la divulgación.

La difusión científica se da internamente, en ámbitos científicos y académicos, cuando los investigadores cumplen con sus pares comunicando sus hallazgos. Para medir la calidad de los aportes y el alcance de la difusión existen indicadores ampliamente aceptados, como los propuestos por Scopus, Web of Science y Scimago (González et al., 2019; Chinchilla., 2011).

Por su parte, la divulgación de la ciencia cumple otro objetivo de la actividad científica; según postulan Grillo et al. (2016), la popularización del conocimiento (divulgación) permite el diálogo dinámico e interdependiente entre ciencia y sociedad, se da fuera de la academia y facilita la comprensión holística del mundo ante la población en general. Estas métricas nacen gracias al crecimiento de herramientas y comunidades en línea, se aplican nuevos filtros para medir el impacto de la producción científica en la sociedad (Priem et al., 2010).

Si bien ambos objetivos de comunicación son imprescindibles (difusión y divulgación), este estudio se enfoca en la difusión de nuevo conocimiento a partir de revistas científicas. En este sentido, el objetivo de este estudio es analizar la difusión de conocimiento desde revistas científicas de alto impacto especializadas en Marketing. 


\section{MÉTODO}

La investigación se desarrolló con el propósito de obtener información exhaustiva de revistas científicas indizadas en Scopus especializadas en la subcategoría Marketing, la información se obtuvo del portal Scimago Journal \& Country Rank y la base de datos de Elsevier. Con corte transversal se recolectó información durante abril y mayo 2021, el estudio se enfocó principalmente en analizar las estadísticas del año 2020, sin embargo, algunos indicadores recogen datos a partir de 2015.

Primero, se realizó un estudio del total de revistas científicas procedentes de todos los países del mundo consideradas en la lista 2020 de Scimago Journal \& Country Rank (SCImago, 2021), registradas en la subcategoría "marketing" de la categoría "negocios, administración y contabilidad". Cumpliendo estos criterios, el total de revistas científicas estudiadas fue 172 , quedando fuera del universo de interés: revistas de negocio, serie de libros, conferencias y congresos. Ver Figura 1.

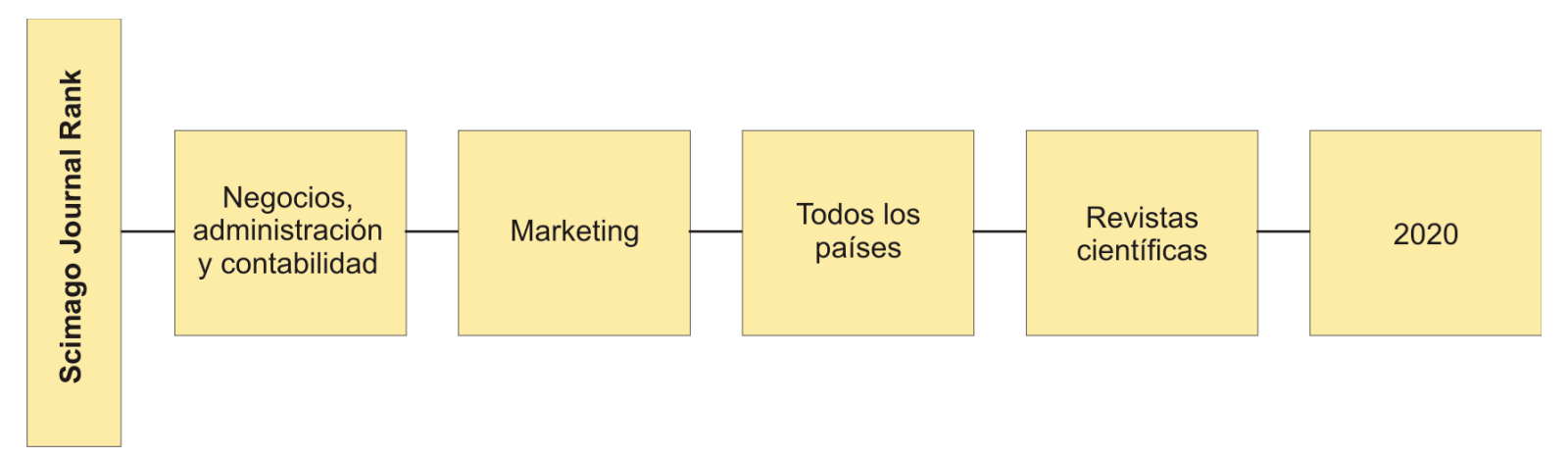

Figura 1. Criterios de selección de la información.

El estudio siguió el criterio expuesto en la figura 1: clasificación de revistas Scimago 2020, subcategoría Marketing, todos los países, solo revistas científicas. Se realizaron cuatro análisis.

Primero, según: región, país, cantidad de revistas por cuartil, tipo de acceso, acreditaciones en SciELO y WoS. El segundo análisis estuvo enfocado en describir la cantidad y participación porcentual de: revistas; documentos publicados en el 2020 (incluyendo documentos citables y no citables); documentos de los últimos 3 años (publicados entre 2017 - 2019, citables y no citables); referencias 2020 (incluye todas las referencias bibliográficas de las revistas) y; citas en los últimos 3 años (citas en 2020 a documentos publicados en entre 2017-2019).

En tercer lugar, se analizaron las 10 revistas con mayor índice SJR. Finalmente, las 23 revistas de acceso abierto. En ambos casos los indicadores considerados fueron: índice SJR (promedio de citas recibidas el 2020 por artículo publicado entre 2017 y 2019); citas últimos 3 años (cantidad de publicaciones entre 2017-2019); índice h (h artículos con al menos h citas cada uno); citas/doc 2 años (promedio de citas recibidas en el 2020 por documento 
publicados entre 2018 y 2019); referencias 2020 (promedio de referencias expuesta en artículos publicos en 2020).

Posteriormente, en busca de información más específica referente a autores y artículos publicados, se consultó la base de datos de Elsevier (Elsevier, 2021). Se crearon 3 grupos: Todo Scopus (5 revistas con mayor índice SJR), Solo acceso abierto (5 revistas con mayor índice SJR) y Solo SciELO (el total de revistas). Esta categorización permitió analizar las siguientes métricas del periodo 2015-2019: autores de mayor impacto, instituciones de afiliación más recurrentes, países a los que están afiliadas las instituciones e instituciones financiadoras.

Es importante aclara que las métricas asumidas consideraron el impacto de la difusión científica, dejando para un próximo estudio el impacto de la divulgación científica.

\section{RESULTADOS}

e presenta un análisis del impacto de la difusión científica especializada en Marketing que considera como objeto de estudio las revistas científicas indizadas en Scopus. Primero, se caracterizó 172 revistas científicas procedentes de todos los países del mundo, consideradas en la lista 2020 de Scimago Journal \& Country Rank. Segundo, se mostró la selección de 13 revistas representativas de 3 grupos: Todo Scopus, Solo acceso abierto y Solo SciELO, para analizar: autores de mayor impacto, temas abordados, instituciones de afiliación, instituciones financiadoras y países recurrentes. El diagnóstico partió por analizar las métricas del año 2020 de revistas científicas indizadas en Scopus, considerando la clasificación de países regional de Scimago: Asia, Europa Oriental, Norte América, Europa Occidental, África, África Medio Oriente, Latinoamérica, Oriente Medio y Región Pacífica (ver Tabla 1).

Tabla 1. Cantidad de revistas según mejor cuartil JRS, tipo de acceso y acreditaciones.

\begin{tabular}{|c|c|c|c|c|c|c|c|c|c|}
\hline \multirow{2}{*}{ Región } & \multicolumn{4}{|c|}{ Cantidad de revistas } & \multirow{2}{*}{ Total } & \multirow{2}{*}{$\begin{array}{c}\% \text { de } \\
\text { participación }\end{array}$} & \multicolumn{3}{|c|}{$\begin{array}{l}\text { Tipo de acceso y } \\
\text { acreditaciones }\end{array}$} \\
\hline & Q1 & Q2 & Q3 & Q4 & & & $\begin{array}{l}\text { Acceso } \\
\text { abierto }\end{array}$ & SciELO & Wos \\
\hline Europa Occidental & 26 & 34 & 19 & 13 & 92 & 53.5 & 6 & & 70 \\
\hline Norte América & 21 & 11 & 18 & 9 & 59 & 34.3 & 2 & & 44 \\
\hline Europa del Este & & & 6 & 1 & 7 & 4.1 & 6 & & 4 \\
\hline Región Asiática & & 1 & 3 & 3 & 7 & 4.1 & 4 & & 2 \\
\hline Latinoamérica & & & & 3 & 3 & 1.7 & 3 & 3 & 2 \\
\hline Región Pacífica & & 1 & & 2 & 3 & 1.7 & 1 & & 1 \\
\hline Oriente Medio & & & & 1 & 1 & 0.6 & 1 & & \\
\hline Total & 47 & 47 & 46 & 32 & 172 & 100.0 & 23 & 3 & 123 \\
\hline
\end{tabular}

Fuente. Con datos de SCImago (2021). 
La Tabla 1 permite comprender, en términos generales, la participación regional actual de las revistas científicas especializadas en Marketing. Los datos recogen las revistas vigentes al 2020, al lado izquierdo se hace una clasificación según el mejor cuartil SJR, expresando la cantidad de revistas Q1, Q2, Q3 y Q4. Asimismo, se presenta la suma total de revistas por región y su respectiva participación porcentual. Se presenta también la cantidad de revistas de acceso abierto. Y, en las últimas 2 columnas se especifica la cantidad de revistas indizadas en SciELO y WoS.

El $88 \%$ de las revistas de impacto internacional están en Europa Occidental y Norte América; asimismo, el 100\% de las revistas Q1 están concentradas en estas dos regiones. Europa del Este y Región Asiática contribuyen con 4.1\% cada una. Latinoamérica y Región Pacífica aportan solamente con $1.7 \%$ cada una. Oriente Medio contribuye con el $0.6 \%$ restante. África y África Medio Oriente no cuentan con revistas en esta subcategoría. Las revistas de acceso abierto corresponden solamente al 13.4\% del total de revistas, 1.7\% están indizadas en SciELO y $71.5 \%$ en WoS. El 17\% de las revistas Q1 es de acceso abierto.

En la Tabla 2 se profundiza el análisis especificando los países de procedencia y otras métricas.

Tabla 2. Revistas, publicaciones, referencias y citas.

\begin{tabular}{|c|c|c|c|c|c|c|c|c|c|c|}
\hline \multirow[t]{2}{*}{ Región/país } & \multicolumn{2}{|c|}{ Revistas } & \multicolumn{2}{|c|}{$\begin{array}{l}\text { Total docs. } \\
(2020)\end{array}$} & \multicolumn{2}{|c|}{$\begin{array}{c}\text { Total docs. } \\
\text { (3 últimos años) }\end{array}$} & \multicolumn{2}{|c|}{ Total refs. } & \multicolumn{2}{|c|}{$\begin{array}{l}\text { Total citas } \\
\text { (3 años) }\end{array}$} \\
\hline & Cantidad & $\%$ & Cantidad & $\%$ & Cantidad & $\%$ & Cantidad & $\%$ & Cantidad & $\%$ \\
\hline Región Asiática & 7 & 4.1 & 208 & 2.0 & 757 & 3.3 & 8920 & 1.3 & 1672 & 0.9 \\
\hline India & 1 & 0.6 & 34 & 0.3 & 145 & 0.6 & 1218 & 0.2 & 254 & 0.1 \\
\hline Filipinas & 1 & 0.6 & 25 & 0.2 & 98 & 0.4 & 868 & 0.1 & 118 & 0.1 \\
\hline Singapur & 1 & 0.6 & 36 & 0.4 & 92 & 0.4 & 1467 & 0.2 & 474 & 0.3 \\
\hline Corea del Sur & 2 & 1.2 & 79 & 0.8 & 346 & 1.5 & 3229 & 0.5 & 496 & 0.3 \\
\hline Taiwán & 2 & 1.2 & 34 & 0.3 & 76 & 0.3 & 2138 & 0.3 & 330 & 0.2 \\
\hline Latinoamérica & 3 & 1.7 & 100 & 1.0 & 408 & 1.8 & 4859 & 0.7 & 382 & 0.2 \\
\hline Brasil & 1 & 0.6 & 38 & 0.4 & 147 & 0.6 & 1440 & 0.2 & 184 & 0.1 \\
\hline Colombia & 2 & 1.2 & 62 & 0.6 & 261 & 1.2 & 3419 & 0.5 & 198 & 0.1 \\
\hline Oriente Medio & 1 & 0.6 & 0 & 0.0 & 107 & 0.5 & 0 & 0.0 & 80 & 0.0 \\
\hline Irán & 1 & 0.6 & 0 & 0.0 & 107 & 0.5 & 0 & 0.0 & 80 & 0.0 \\
\hline Norte América & 59 & 34.3 & 3558 & 35.1 & 7595 & 33.5 & 242490 & 35.7 & 74894 & 40.0 \\
\hline Estados Unidos & 59 & 34.3 & 3558 & 35.1 & 7595 & 33.5 & 242490 & 35.7 & 74894 & 40.0 \\
\hline Región Pacífica & 3 & 1.7 & 96 & 0.9 & 153 & 0.7 & 7292 & 1.1 & 1008 & 0.5 \\
\hline Australia & 2 & 1.2 & 72 & 0.7 & 122 & 0.5 & 5489 & 0.8 & 950 & 0.5 \\
\hline Nueva Zelanda & 1 & 0.6 & 24 & 0.2 & 31 & 0.1 & 1803 & 0.3 & 58 & 0.0 \\
\hline
\end{tabular}




\begin{tabular}{lcccccccccc}
\hline \multirow{2}{*}{ Región/país } & \multicolumn{2}{c}{ Revistas } & \multicolumn{3}{c}{$\begin{array}{c}\text { Total docs. } \\
\text { (2020) }\end{array}$} & \multicolumn{2}{c}{ Total docs. } & \multicolumn{2}{c}{ (3 últimos años) } & \multicolumn{2}{c}{ Total refs. } & \multicolumn{3}{c}{$\begin{array}{c}\text { Total citas } \\
\text { (3 años) }\end{array}$} \\
& Cantidad & $\%$ & Cantidad & $\%$ & Cantidad & $\%$ & Cantidad & $\%$ & Cantidad & \% \\
\hline $\begin{array}{l}\text { Europa } \\
\text { Occidental }\end{array}$ & $\mathbf{9 2}$ & $\mathbf{5 3 . 5}$ & $\mathbf{5 8 7 2}$ & $\mathbf{5 7 . 9}$ & $\mathbf{1 2 8 1 9}$ & $\mathbf{5 6 . 6}$ & $\mathbf{4 0 1 8 0 4}$ & $\mathbf{5 9 . 1}$ & $\mathbf{1 0 7 0 0 7}$ & $\mathbf{5 7 . 2}$ \\
Bélgica & 1 & 0.6 & 63 & 0.6 & 119 & 0.5 & 1765 & 0.3 & 166 & 0.1 \\
Francia & 1 & 0.6 & 8 & 0.1 & 58 & 0.3 & 185 & 0.0 & 10 & 0.0 \\
Alemania & 6 & 3.5 & 183 & 1.8 & 366 & 1.6 & 9898 & 1.5 & 1690 & 0.9 \\
Grecia & 1 & 0.6 & 112 & 1.1 & 169 & 0.7 & 3514 & 0.5 & 252 & 0.1 \\
Países Bajos & $\mathbf{6}$ & $\mathbf{3 . 5}$ & $\mathbf{4 0 7}$ & $\mathbf{4 . 0}$ & $\mathbf{8 5 4}$ & $\mathbf{3 . 8}$ & $\mathbf{2 7 5 6 1}$ & $\mathbf{4 . 1}$ & $\mathbf{1 0 2 3 8}$ & $\mathbf{5 . 5}$ \\
España & 2 & 1.2 & 42 & 0.4 & 121 & 0.5 & 3167 & 0.5 & 1432 & 0.8 \\
Suiza & 3 & 1.7 & 133 & 1.3 & 424 & 1.9 & 5367 & 0.8 & 801 & 0.4 \\
\hline
\end{tabular}

Fuente. Con datos de SCImago (2021).

La Tabla 2 detalla región y país, se expone la cantidad y participación porcentual de: revistas; documentos publicados en el 2020 (incluyendo documentos citables y no citables); documentos de los últimos 3 años (publicados entre 2017 - 2019, citables y no citables); referencias 2020 (Incluye todas las referencias bibliográficas de las revistas) y; citas en los últimos 3 años (citas recibidas en 2020 a los documentos publicados en entre 2017-2019).

Nótese que si bien la región Europa Occidental tiene el 53.5\% de revistas: publicó el $57.9 \%$ de la producción intelectual en el 2020, el 56.6\% durante 2017-2019, tiene el 59.1\% del total de referencias y ha conseguido el $57.2 \%$ de citas en 2020 a documentos publicados durante 2017-2019. Actualmente, en esta región, son 8 los países/naciones que tienen revistas especializadas en Marketing: Reino Unido, Países Bajos, Alemania, Suiza, España, Bélgica, Francia y Grecia. Reino Unido tiene la mayor participación a nivel mundial: 41.9\% de las revistas y $49.4 \%$ de las citas recibidas en 2020 a nivel mundial. Países Bajos y Alemania tienen la misma cantidad de revistas (3.5\% del total a nivel mundial); sin embargo, el impacto que han logrado es muy diferente: las publicaciones desde Países Bajos han obtenido en 2020 al $5 \%$ de las citas a nivel mundial, en cambio las de Alemania solamente $0.9 \%$.

En Norte América, elúnico país que posee revistas de impacto internacional especializadas en Marketing es Estados Unidos: tiene el 34.3\% de revistas, publicó el $35.1 \%$ de la producción intelectual en el 2020, el 33.5\% durante 2017-2019, posee el 35.7\% del total de referencias y ha conseguido el $40 \%$ de citas en 2020 a documentos publicados durante 2017-2019.

Latinoamérica aporta el $1.7 \%$ de las revistas, ha publicado en 2020 sólo $1 \%$ del total mundial ( $0.6 \%$ por Brasil y 0.4 por Colombia); tiene el $1.8 \%$ de las referencias realizadas en 2020 a documentos publicados entre $2017-2019$ (1.2\% a revistas de Colombia y $0.6 \%$ de Brasil).

En Europa del Este resalta Ucrania, que tiene el 1.2\% de los documentos publicados en 2020 a nivel mundial, sin embargo, las citas recibidas son bajas con relación a su participación (0.3\%). Algo similar acurre en la Región Asiática, dónde Taiwán tiene el 1.2\% de la publicación mundial pero solo recibe el $0.2 \%$ de referencias. 
Tabla 3. Revistas Q1 a nivel mundial - Selección de las 10 primeras.

\begin{tabular}{|c|c|c|c|c|c|c|}
\hline Etiquetas de fila & SJR & Índice H & $\begin{array}{l}\text { Documentos } \\
\text { citables } \\
\text { (3años) }\end{array}$ & $\begin{array}{l}\text { Citas / Doc. } \\
\quad 2 \text { años }\end{array}$ & $\begin{array}{l}\text { Ref. / } \\
\text { Doc. }\end{array}$ & País \\
\hline Journal of Consumer Research & 8.916 & 179 & 186 & 13.74 & 58.24 & $\begin{array}{l}\text { Reino } \\
\text { Unido }\end{array}$ \\
\hline Journal of Marketing & 7.799 & 243 & 146 & 19.31 & 73.15 & $\begin{array}{l}\text { Estados } \\
\text { Unidos }\end{array}$ \\
\hline Journal of Marketing Research & 6.321 & 171 & 183 & 10.48 & 57.36 & $\begin{array}{l}\text { Estados } \\
\text { Unidos }\end{array}$ \\
\hline Marketing Science & 5.938 & 127 & 153 & 8.29 & 43.13 & $\begin{array}{l}\text { Estados } \\
\text { Unidos }\end{array}$ \\
\hline $\begin{array}{l}\text { Journal of the Academy of Marketing } \\
\text { Science }\end{array}$ & 5.512 & 170 & 154 & 16.5 & 87.54 & $\begin{array}{l}\text { Estados } \\
\text { Unidos }\end{array}$ \\
\hline Journal of Consumer Psychology & 4.433 & 110 & 144 & 6.81 & 58.38 & $\begin{array}{l}\text { Estados } \\
\text { Unidos }\end{array}$ \\
\hline $\begin{array}{l}\text { Journal of Public Administration } \\
\text { Research and Theory }\end{array}$ & 4.154 & 112 & 107 & 11.7 & 71.54 & $\begin{array}{l}\text { Reino } \\
\text { Unido }\end{array}$ \\
\hline Journal of Supply Chain Management & 3.75 & 92 & 54 & 15.57 & 96.07 & $\begin{array}{l}\text { Estados } \\
\text { Unidos }\end{array}$ \\
\hline $\begin{array}{l}\text { International Journal of Research in } \\
\text { Marketing }\end{array}$ & 3.725 & 102 & 129 & 10.3 & 74.93 & Holanda \\
\hline Journal of World Business & 3.607 & 112 & 168 & 17.49 & 109.67 & $\begin{array}{l}\text { Estados } \\
\text { Unidos }\end{array}$ \\
\hline
\end{tabular}

Fuente. Con datos de SCImago (2021).

Existen 47 revistas científicas de categoría Q1 especializadas en Marketing, en la tabla 3 se han escogido las 10 mejores considerando estadísticas del 2020. La revista del Reino Unido Journal of Consumer Research es la primera de la lista, tiene un índice SJR de 8.916, es decir en promedio durante el año 2020 ha recibido aproximadamente 9 citas por artículo publicado entre 2017 y 2019 (durante esos 3 años publicó 186 actículos citables). Esta revista tiene un índice h de 179, lo que significa que tiene 179 artículos con al menos 179 citas cada uno; durante 2020 ha recibido en promedio 13.74 citas por documento publicado entre 2018 y 2019; los artículos publicados en 2020 han recibido en promedio 58.24 citas. La revista estadounidense Journal of Marketing tiene 243 artículos citados al menos 243 veces (el mayor índice h de la lista); durante 2020 ha recibido en promedio 19.31 citas por documento publicado entre 2018 y 2019; los artículos publicados en 2020 han recibido en promedio 73.15 citas. La última revista de la lista, Journal of World Business, ha recibido la mayor cantidad de citas en 2020 por artículos publicados entre 2018 y 2019 (109.67 ref/doc).

Según procedencia: 7 revistas son de Estados Unididos, 2 del Reino Unido y 1 de Holanda. Todas se publican en ingles, tienen acceso restringido y están indizadas en WoS. 
Tabla 4. Revistas acceso abierto a nivel mundial.

\begin{tabular}{|c|c|c|c|c|c|c|}
\hline Etiquetas de fila & $\begin{array}{l}\text { Suma } \\
\text { de SJR }\end{array}$ & Índice H & $\begin{array}{l}\text { Documentos } \\
\text { citables } \\
\text { (3años) }\end{array}$ & $\begin{array}{l}\text { Citas / } \\
\text { Doc. } \\
2 \text { años }\end{array}$ & $\begin{array}{l}\text { Ref. / } \\
\text { Doc. }\end{array}$ & País \\
\hline Journal of Innovation and Knowledge & 1720 & 20 & 70 & 21.68 & 67.51 & Holanda \\
\hline $\begin{array}{l}\text { European Research on Management } \\
\text { and Business Economics }\end{array}$ & 1024 & 18 & 63 & 12.69 & 55.95 & España \\
\hline Spanish Journal of Marketing - ESIC & 0.796 & 13 & 58 & 8.23 & 96.8 & España \\
\hline $\begin{array}{l}\text { European Journal of Management } \\
\text { and Business Economics }\end{array}$ & 0.691 & 16 & 66 & 5.63 & 53.12 & $\begin{array}{l}\text { Reino } \\
\text { Unido }\end{array}$ \\
\hline International Journal of Design & 0.51 & 40 & 52 & 4.51 & 64.04 & Taiwán \\
\hline Fashion and Textiles & 0.457 & 13 & 89 & 5.09 & 40.75 & Singapur \\
\hline Organizacija & 0.398 & 8 & 64 & 3.79 & 56.32 & Eslovenia \\
\hline $\begin{array}{l}\text { Transformations in Business and } \\
\text { Economics }\end{array}$ & 0.346 & 20 & 262 & 3.51 & 48.84 & Lituania \\
\hline Cogent Business and Management & 0.345 & 16 & 349 & 4.06 & 69.26 & $\begin{array}{l}\text { Reino } \\
\text { Unido }\end{array}$ \\
\hline $\begin{array}{l}\text { Real Estate Management and } \\
\text { Valuation }\end{array}$ & 0.296 & 8 & 113 & 2.1 & 36.91 & Polonia \\
\hline Innovative Marketing & 0.239 & 5 & 55 & 2.8 & 48.02 & Ucrania \\
\hline Trziste & 0.229 & 7 & 36 & 0.79 & 50.96 & Croacia \\
\hline $\begin{array}{l}\text { Journal of Eastern European and } \\
\text { Central Asian Research }\end{array}$ & 0.223 & 5 & 61 & 1.43 & 35.18 & $\begin{array}{l}\text { Estados } \\
\text { Unidos }\end{array}$ \\
\hline Banks and Bank Systems & 0.194 & 16 & 210 & 2.5 & 39.17 & Ucrania \\
\hline Journal of Distribution Science & 0.19 & 10 & 323 & 1.77 & 41.03 & $\begin{array}{l}\text { Corea del } \\
\text { Sur }\end{array}$ \\
\hline $\begin{array}{l}\text { RAE Revista de Administracao de } \\
\text { Empresas* }\end{array}$ & 0.183 & 15 & 131 & 1.1 & 37.89 & Brasil \\
\hline $\begin{array}{l}\text { Journal of International Logistics and } \\
\text { Trade }\end{array}$ & 0.161 & 3 & 21 & 0.78 & 40.25 & $\begin{array}{l}\text { Corea del } \\
\text { Sur }\end{array}$ \\
\hline Innovar* & 0.156 & 11 & 129 & 0.63 & 54.25 & Colombia \\
\hline $\begin{array}{l}\text { EAl Endorsed Transactions on Energy } \\
\text { Web }\end{array}$ & 0.145 & 6 & 118 & 1.42 & 28.02 & Bélgica \\
\hline Estudios Gerenciales* & 0.144 & 12 & 116 & 0.55 & 56.1 & Colombia \\
\hline $\begin{array}{l}\text { Journal of Food Distribution } \\
\text { Research }\end{array}$ & 0.133 & 1 & 16 & 0.63 & 23.37 & $\begin{array}{l}\text { Estados } \\
\text { Unidos }\end{array}$ \\
\hline $\begin{array}{l}\text { International Journal of Manage- } \\
\text { ment and Business Research }\end{array}$ & 0.125 & 7 & 107 & 0.76 & 0 & Irán \\
\hline $\begin{array}{l}\text { Journal of Empirical Generalisations } \\
\text { in Marketing Science }\end{array}$ & 0.113 & 11 & 3 & 0 & 20 & Australia \\
\hline
\end{tabular}

Nota: *Revistas indexadas en SciELO

Fuente. Con datos de SCImago (2021). 
La Tabla 4, presenta todas las revistas de acceso abierto indizadas en Scopus. Las dos primeras de la lista son categoría Q1 y están indizadas en WoS: Journal of Innovation and Knowledge (Holandesa) y European Research on Management and Business Economics (Española). La revista Transformations in Business and Economics (Lituania) tiene, junto con la holandesa antes citada, el mayor índice h del grupo (h20). La revista del Reino Unido Cogent Business and Management, es la que más artículos científicos ha publicado durante 2017-2019 (349).

Las revistas indizadas en SciELO son: RAE Revista de Administracao de Empresas (Brasil), Innovar (Colombia) y Estudios Gerenciales (Colombia).

Según procedencia, los países que tienen dos revistas son: Reino Unido, Estados Unididos, España, Ucrania, Corea del Sur y Colombia. Los países que tienen una revista son: Holanda, Taiwán, Singapur, Eslovenia, Lituania, Polonia, Croacia, Brasil, Bélgica, Irán y Australia.

En las Tablas 5, 6, 7 y 8 se presenta un análisis de los autores, instituciones de afiliación, países de afiliación e instituciones financiadoras. Se consideraron en 3 grupos las revistas de mayor índice SJR de: Todo Scopus, Sólo acceso abierto y Solo SciELO: El primer grupo, Todo Scopus, enuncia los resultados más recurrentes en las revistas: Journal of Consumer Research, Journal of Marketing, Journal of Marketing Research, Marketing Science y Journal of the Academy of Marketing Science. El segundo grupo, Solo acceso abierto, incluye los resultados más recurrentes de las revistas: Journal of Innovation and Knowledge, European Research on Management and Business Economics, Spanish Journal of Marketing - ESIC, European Journal of Management and Business Economics e International Journal of Design. En el tercer grupo, Solo SciELO, se incluye las únicas 3 revistas indizadas en SciELO: RAE Revista de Administracao de Empresas, Innovar y Estudios Gerenciales.

Tabla 5. Autores recurrentes de mayor índice SJR (2016-2020).

\begin{tabular}{lclclc}
\hline \multicolumn{2}{c}{ Todo Scopus } & \multicolumn{2}{c}{ Solo acceso abierto } & \multicolumn{2}{c}{ Solo Scielo } \\
\multicolumn{1}{c}{ Autor } & Artículos & \multicolumn{1}{c}{ Autor } & Artículos & \multicolumn{1}{c}{ Autor } & Artículos \\
\hline Kumar, V. & 38 & Desmet, P.M.A. & 12 & Tonelli, M.J. & 19 \\
Palmatier, R.W. & 19 & Hekkert, P. & 11 & Zambaldi, F. & 17 \\
Chintagunta, P.K. & 14 & Schifferstein, H.N.J. & 11 & Alonso, J.C. & 9 \\
Grewal, R. & 14 & Bigne, E. & 8 & Villegas, M.G. & 9 \\
Dahl, D.W. & 13 & Karana, E. & 7 & Milanesi, G.S. & 8 \\
Kannan, P.K. & 13 & Mugge, R. & 6 & Rodríguez, V.M.C. & 7 \\
Mittal, V. & 13 & Rita, P. & 6 & Dabos, G.E. & 6 \\
Sridhar, S. & 13 & Chen, L.L. & 5 & Alcadipani, R. & 4 \\
Sudhir, K. & 12 & Delgado-Ballester, E. & 5 & Arango, A.M.A. & 4 \\
Bagchi, R. & 11 & Nam, T.J. & 5 & Ayala, A.H. & 4 \\
\hline
\end{tabular}

Fuente. Con datos de Elsevier (2021). 
La Tabla 5, presenta 30 autores destacados en la subcategoría Marketing, puntualizando en la cantidad de artículos publicados durante el periodo 2016-2020 en las revistas de mayor índice SJR. A continuación, se caracteriza a los 5 autores más sobresalientes.

Resalta el autor indú Vikas Kumar, con: un total de 38 artículos publicados durante el periodo de estudio en las 5 mejores revistas de Scopus, h59, 211 artículos de impacto internacional, a la fecha ha sido citado por 9724 documentos. Entre 2015 y 2019 ha publicado: 10 documentos sobre Rotación de clientes, Valor de vida del cliente y Gestión de relaciones con el cliente; 8 documentos sobre Boca a boca electrónica, Reseñas en línea y Comunidad de marca; 4 sobre Promociones de precios, Cupones y Reembolsos. Su artículo más sonbresaliente tiene 774 citas "On the profitability of long-life customers in a noncontractual setting: An empirical investigation and implications for marketing" (Reinartz \& Kumar, 2000) y el artículo más reciente se denomina "Chief marketing officers 'discretion and firms' internationalization: An empirical investigation” (Kumar et al., 2021).

Por su parte, el estadounidense Robert Palmatier, con: un total de 19 artículos publicados entre 2016 y 2021 en las 5 mejores revistas de Scopus, h36, 80 artículos de impacto internacional, ha sido citado por 5159 documentos. Entre 2015 y 2019 publicó: 9 documentos sobre Relaciones comprador-proveedor, Oportunismo y Gobernanza relacional; 4 docmuentos sobre Boca a boca electrónica, Reseñas en línea y Comunidad de marca; 3 sobre promociones de Investigación de mercado, Marketing y Publicaciones académicas. Su artículo "Factors influencing the effectiveness of relationship marketing: A meta-analysis" (Palmatier et al., 2006) tiene 1538 citas y el más reciente se denomina "Commentary: Opportunities and challenges of technology in relationship marketing" (Steinhoff \& Palmatier, 2021).

El investigador indú Pradeep Chintagunta, con: un total de 14 artículos publicados durante el periodo de estudio en las 5 mejores revistas de Scopus, h42, 117 artículos de impacto internacional, a la fecha ha sido citado por 4265 documentos. Entre 2015 y 2019 ha publicado: 6 documentos sobre Elección discreta dinámica; Modelo estructural dinámico; Juegos discretos; 2 documentos sobre Promociones de precios, Cupones y Reembolsos; 2 sobre Búsqueda de consumidores; Dispersión de precios; Shopbots. Su artículo más sonbresaliente tiene 452 citas "The effects of online user reviews on movie box office performance: Accounting for sequential rollout and aggregation across local markets" (Chintagunta et al., 2010) y el más reciente se denomina "Social Media, Influencers, and Adoption of an Eco-Friendly Product: Field Experiment Evidence from Rural China" (Zhang et al., 2021).

El autor estadounidense Dhruv Grewal, con: un total de 14 artículos publicados entre 2016 y 2021 en las 5 mejores revistas de Scopus, h60, 175 artículos de impacto internacional, citado por 13799 documentos. Entre 2015 y 2019 ha publicado: 4 documentos sobre Boca a boca electrónica, Reseñas en línea y Comunidad de marca; 4 documentos sonbre Promociones de precios, Cupones y Reembolsos; 3 sobre Precio de referencia, Precios por comportamiento y Descuentos. Su artículo que ha alcanzado mayor impacto a la fecha tiene 1539 citas "Factors influencing the effectiveness of relationship marketing: A meta-analysis" (Palmatier et al., 2006) y el más reciente se denomina "Navigating the Retailing Frontier through Academic and Practitioner Collaboration” (Gauri \& Grewal, 2021). 
El autor estadounidense Darren Dahl, con un total de 13 artículos publicados entre 2016 y 2021 en las 5 mejores revistas de Scopus, h42, 104 artículos de impacto internacional, citado por 4535 documentos. Entre 2015 y 2019 ha publicado: 2 documentos sobre Recuperación del servicio; Comportamiento de quejas; Justicia percibida; 1 sobre Boca a boca electrónica; Reseñas en línea; Comunidad de marca; 1 sobre Colocación de marca; Publicidad de alimentos; Modelo de conocimiento de persuasión. Su artículo que ha alcanzado mayor impacto a la fecha tiene 378 citas "The influence and value of analogical thinking during new product ideation" (Dahl \& Moreau, 2002) y el más reciente se denomina "Marketers Project Their Personal Preferences onto Consumers: Overcoming the Threat of Egocentric Decision Making" (Herzog et al., 2021).

Tabla 6. Instituciones de afiliación recurrentes

\begin{tabular}{|c|c|c|c|c|c|}
\hline \multicolumn{2}{|l|}{ Todo Scopus } & \multicolumn{2}{|l|}{ Solo acceso abierto } & \multicolumn{2}{|l|}{ Solo SciELO } \\
\hline $\begin{array}{l}\text { Institución de } \\
\text { afiliación }\end{array}$ & Total & $\begin{array}{l}\text { Institución de } \\
\text { afiliación }\end{array}$ & Total & $\begin{array}{l}\text { Institución de } \\
\text { afiliación }\end{array}$ & Total \\
\hline Texas A\&M University & 71 & $\begin{array}{l}\text { Delft University of } \\
\text { Technology }\end{array}$ & 62 & Fundacao Getulio Vargas & 66 \\
\hline $\begin{array}{l}\text { Georgia State } \\
\text { University }\end{array}$ & 61 & University of Valencia & 44 & Universidad Icesi & 65 \\
\hline $\begin{array}{l}\text { Terry College of } \\
\text { Business }\end{array}$ & 54 & $\begin{array}{l}\text { Technische Universiteit } \\
\text { Eindhoven }\end{array}$ & 25 & $\begin{array}{l}\text { Universidad Nacional de } \\
\text { Colombia }\end{array}$ & 43 \\
\hline University of Georgia & 49 & University de Sevilla & 21 & Universidad del Valle, Cali & 38 \\
\hline $\begin{array}{l}\text { University of } \\
\text { Washington }\end{array}$ & 49 & $\begin{array}{l}\text { Korea Advanced Institute of } \\
\text { Science \& Technology }\end{array}$ & 16 & Universidad EAFIT & 26 \\
\hline $\begin{array}{l}\text { J. Mack Robinson } \\
\text { College of Business }\end{array}$ & 48 & $\begin{array}{l}\text { National Taiwan University } \\
\text { of Science and Technology }\end{array}$ & 14 & $\begin{array}{l}\text { Universidade de Sao } \\
\text { Paulo - USP }\end{array}$ & 22 \\
\hline Mays Business School & 48 & Aalto University & 13 & University de Sevilla & 18 \\
\hline $\begin{array}{l}\text { University of Maryland, } \\
\text { College Park UMD }\end{array}$ & 41 & $\begin{array}{l}\text { Hong Kong Polytechnic } \\
\text { University }\end{array}$ & 13 & $\begin{array}{l}\text { Pontificia Universidad } \\
\text { Javeriana }\end{array}$ & 16 \\
\hline $\begin{array}{l}\text { Foster School of } \\
\text { Business }\end{array}$ & 40 & Universidad de Zaragoza & 13 & Tecnológico de Monterrey & 16 \\
\hline $\begin{array}{l}\text { Indiana University } \\
\text { Bloomington }\end{array}$ & 40 & $\begin{array}{l}\text { Universidad de } \\
\text { Extremadura }\end{array}$ & 12 & Universidad de Antioquia & 16 \\
\hline
\end{tabular}

Fuente. Con datos de Elsevier (2021). 
LaTabla 6, presenta 29 instituciones de afiliación destacadas en la subcategoría Marketing, puntualizando en la cantidad total de artículos publicados por sus afiliados durante el periodo 2016-2019. La institución que mayor cantidad registra es la norteamericana Texas A\&M University (71 investigaciones), seguida por la brasilera Fundacao Getulio Vargas (66), la colombiana Universidad Icesi (65), Delft University of Technology de los Paises Bajos (62) y Georgia State University de Estados Unidos (61). La única universidad que muestra afiliación en las mejores revistas de acceso abierto y en SciELO es la Universidad de Sevilla (39 investigaciones en total).

Tabla 7. Países de origen de las instituciones de afiliación.

\begin{tabular}{lccclc}
\hline $\begin{array}{c}\text { Todo Scopus } \\
\text { País de origen del } \\
\text { documento }\end{array}$ & Total & \multicolumn{1}{c}{$\begin{array}{c}\text { Solo acceso abierto } \\
\text { País de origen del } \\
\text { documento }\end{array}$} & Total & $\begin{array}{c}\text { Solo SciELo } \\
\text { País de origen del } \\
\text { documento }\end{array}$ & Total \\
\hline Estados Unidos & 1076 & España & 182 & Colombia & 365 \\
Alemania & 157 & Países Bajos & 95 & Brasil & 222 \\
porcelana & 116 & Reino Unido & 67 & España & 150 \\
Reino Unido & 110 & Estados Unidos & 56 & México & 105 \\
Canadá & 93 & Portugal & 42 & Chile & 64 \\
Países Bajos & 89 & Taiwán & 36 & Argentina & 45 \\
Australia & 67 & Corea del Sur & 33 & Estados Unidos & 40 \\
India & 60 & Suecia & 33 & Perú & 20 \\
Hong Kong & 43 & Dinamarca & 24 & Venezuela & 17 \\
Francia & 37 & Italia & 23 & Ecuador & 14 \\
\hline
\end{tabular}

Fuente. Con datos de Elsevier (2021).

La Tabla 7, presenta 26 países de origen destacados en la subcategoría Marketing, puntualizando en la cantidad total de artículos publicados durante el periodo 2016-2019. Resaltan Estados unidos con participación en los 3 grupos, Reino Unidos y Países Bajos con participación en Todo Scopus y Solo acceso abierto y España por participación en Solo acceso abierto y Solo SciELO. En el periodo de estudio: 1172 artículos están afiliados a instituciones de Estados Unidos, 365 a instituciones de Colombia, 332 a instituciones Españolas, 222 artículos a instituciones de Brasil, 184 a Países Bajos, 177 a Reino Unido, entre los más sobresalientes. 
Tabla 8. Instituciones financiadoras recurrentes.

\begin{tabular}{|c|c|c|c|c|c|}
\hline Todo Scopus & & Solo acceso abierto & & Solo SciELO & \\
\hline Intitución financiadora & Total & Intitución financiadora & Total & Intitución financiadora & Total \\
\hline $\begin{array}{l}\text { National Natural } \\
\text { Science Foundation of } \\
\text { China }\end{array}$ & 65 & $\begin{array}{l}\text { Ministerio de Economía y } \\
\text { Competitividad (España) }\end{array}$ & 19 & $\begin{array}{l}\text { Conselho Nacional } \\
\text { de Desenvolvimento } \\
\text { Científico e Tecnológico }\end{array}$ & 8 \\
\hline $\begin{array}{l}\text { Marketing Science } \\
\text { Institute }\end{array}$ & 43 & European Commission & 18 & $\begin{array}{l}\text { Coordenação de } \\
\text { Aperfeiçoamento de } \\
\text { Pessoal de Nível Superior }\end{array}$ & 8 \\
\hline $\begin{array}{l}\text { Social Sciences and } \\
\text { Humanities Research } \\
\text { Council of Canada }\end{array}$ & 38 & University of Valencia & 15 & $\begin{array}{l}\text { Universidad Nacional de } \\
\text { Colombia }\end{array}$ & 6 \\
\hline Government of Canada & 34 & $\begin{array}{l}\text { European Regional } \\
\text { Development Fund }\end{array}$ & 10 & $\begin{array}{l}\text { MinistÃrio da Ciência, } \\
\text { Tecnologia e Inovação }\end{array}$ & 5 \\
\hline University of Chicago & 16 & $\begin{array}{l}\text { Universidade da Beira } \\
\text { Interior }\end{array}$ & 7 & Total & 5 \\
\hline $\begin{array}{l}\text { National Science } \\
\text { Foundation }\end{array}$ & 13 & $\begin{array}{l}\text { Universitat Politécnica de } \\
\text { València }\end{array}$ & 7 & $\begin{array}{l}\text { Consejo Nacional de } \\
\text { Ciencia y Tecnología }\end{array}$ & 4 \\
\hline Cornell University & 11 & European Social Fund & 6 & $\begin{array}{l}\text { Fundação para a Ciência } \\
\text { e a Tecnologia }\end{array}$ & 4 \\
\hline $\begin{array}{l}\text { Deutsche } \\
\text { orschungsgemeinschaft }\end{array}$ & 11 & University of Seville & 6 & $\begin{array}{l}\text { Fondo Nacional de } \\
\text { Desarrollo Científico y } \\
\text { Tecnológico }\end{array}$ & 3 \\
\hline $\begin{array}{l}\text { Nederlandse } \\
\text { Organisatie voor } \\
\text { Wetenschappelijk } \\
\text { Onderzoek }\end{array}$ & 11 & $\begin{array}{l}\text { Directorate-General for } \\
\text { Employment, Social Affairs } \\
\text { and Inclusion }\end{array}$ & 5 & Universidad EAFIT & 3 \\
\hline $\begin{array}{l}\text { Washington University } \\
\text { in St. Louis }\end{array}$ & 11 & $\begin{array}{l}\text { Fundação para a Ciência e } \\
\text { a Tecnologia }\end{array}$ & 5 & $\begin{array}{l}\text { Consejo Nacional de } \\
\text { Rectores }\end{array}$ & 2 \\
\hline
\end{tabular}

Fuente. Con datos de Elsevier (2021).

La Tabla 8, presenta 29 instituciones financiadoras destacadas, puntualizando en la cantidad total de artículos solventados durante el periodo 2016-2019 en las 5 revistas de acceso abierto de mayor índice SJR. La institución con mayor cantidad de registros es National Natural Science Foundation of China fundada en 1986, financió en el periodo de estudio 65 investigaciones. En segundo lugar, está el Marketing Science Institute con base en Estados Unidos que financió 43 investigaciones. La institución canadiense Social Sciences and Humanities Research Council of Canada financió 38 investigaciones, el Gobierno de Canadá financió 34 estudios. El Ministerio de Economía y Competitividad de España financió 19 investigaciones. 


\section{CONCLUSIONES}

egún criterios internacionalmente aceptados, las 172 revistas científicas consideradas en este estudio son las que mayor impacto tienen en términos de difusión de conocimiento especializado en marketing. La producción se caracteriza por estar en inglés y ser de acceso restringido. Destaca Reino Unido con más participación y cuya revista Journal of Consumer Research tiene el mayor impacto a nivel mundial. Estados Unidos es el segundo en participación y tiene siete de las mejores diez revistas. Desde Reino Unido y Estados Unidos se publica tres cuartos de la producción, la relación entre país de procedencia de la revistas y país de afiliación es alta, sin embargo, el país de procedencia de muchos de los autores es otro; dos de los cinco autores más recurrentes provienen de Asia (específicamente de la India). No se han encontrado métricas (internacionalmente aceptadas) sobre la nacionalidad de los principales líderes científicos. En algunos casos, el lugar de nacimiento no figura en los sitios web y redes sociales de los propios autores.

Al comparar el impacto relativo con la participación relativa, destaca Países Bajos por haber obtenido un impacto considerablemente mayor a su participación (cantidad de revistas). De manera inversa, resaltan Alemania, Ucrania y Taiwán por haber generado un impacto considerablemente menor al de su participación.

Existen revistas cuya participación es estratégica para la difusión de conocimiento en países en desarrollo: Journal of Innovation and Knowledge (Holandesa) y European Research on Management and Business Economics (Española). Ambas son de acceso abierto, categoría Q1 y están indizadas en WoS. Asimismo, sobresale la revista Transformations in Business and Economics (Lituania) que tiene, junto con la holandesa antes citada, el mayor índice $h$ del grupo de revistas de acceso abierto. La revista del Reino Unido Cogent Business and Management es la que más artículos científicos ha publicado durante 2017-2019. Finalmente, para la comunidad de investigadores que buscar publicar/investigar en español están las revistas colombianas Innovar y Estudios Gerenciales; la revista RAE Revista de Administracao de Empresas de Brasil publica en portuguez.

Por otra parte, los autores más recurrentes en SciELO no son los más recurrentes en las revistas de mayor impacto de acceso abierto; estos últimos no publican en las 5 revistas de mayor impacto de Scopus. Del total de revistas analizadas, resaltaron los autores hindúes Vikas Kumar y Pradeep Chintagunta, así como los autores estadounidenses Robert Palmatier, Dhruv Grewal y Darren Dahl. Se encontró que las temáticas más estudiadas por estos autores en los últimos seis años son: Boca a boca electrónica, Reseñas en línea y Comunidad de marca y; Promociones de precios, Cupones y Reembolsos. 


\section{REFERENCIAS}

Chinchilla, Z., y De Moya, F. (2011). Open access and Scopus: A new approach to scientific visibility from the standpoint of access. Journal of the American society for information science and technology, 62(6), 1130-1145

Chintagunta, P.; Gopinath, S., y Venkataraman, S. (2010). The effects of online user reviews on movie box office performance: Accounting for sequential rollout and aggregation across local markets. Marketing science, 29(5), 944-957

Dahl, D., y Moreau, P. (2002). The influence and value of analogical thinking during new product ideation. Journal of marketing research, 39(1), 47-60

Elsevier (2021). Elsevier B.V. [Portal]. mayo 2021. De https://www.sciencedirect.com

Espinosa, P. (2019). La difusión del conocimiento (Editorial). Revista Médica del Instituto del Seguro Social. México. Redalyc: http://www. redalyc.org/articulo.oa?id=457761131001

Gauri, D., y Grewal, D. (2021). Navigating the Retailing Frontier through Academic and Practitioner Collaboration. Journal of Retailing, 2021, 97(1), pp. 2-5

González, J.; Díaz, J., y Castro, A. (2019). Análisis de los indicadores de citación de las Revistas Científicas colombianas en el área de Ingeniería. Información tecnológica, 30(2), 293-302

Grillo, S.; Giering, M., y Motta, D. (2016). Perspectivas discursivas da divulgação/ popularização da ciência. Bakhtiniana: Revista de Estudos do Discurso [online]. 2016, v. 11, n. 2 [Accessed 26 May 2021], pp. 3-13. Available from: <https://doi. org/10.1590/2176-457327166>. ISSN 2176-4573. https://doi.org/10.1590/2176457327166.

Hanwell, M.; Curtis, D.; Lonie, D.; Vandermeerschd, T.; Zurek, E., y Hutchison, G. (2012). Avogadro: An advanced semantic chemical editor, visualization, and analysis platform. Journal of CheminformaticsOpen AccessVolume 4, Issue 8 August 2012 Article number 17
Herzog, W.; Hattula, J., y Dahl, D.W. (2021). Marketers Project Their Personal Preferences onto Consumers: Overcoming the Threat of Egocentric Decision Making. Journal of Marketing Research, 2021, 58(3), pp. 456-475

Kumar, V., Borah, S., Sharma, A., Akella, L.Y. (2021) Chief marketing officers' discretion and firms' internationalization: An empirical investigation. Journal of International Business Studies, 2021, 52(3), pp. 363-387

Palmatier, R.; Dant, R.; Grewal, D., y Evans, K. (2006). Factors influencing the effectiveness of relationship marketing: A meta-analysis. Journal of Marketing, 2006, 70(4), pp. 136153

Priem, J.; Taraborelli, D.; Groth, P., y Neylon, C. (2010). Altmetrics: A manifesto, 26 October 2010. http://altmetrics.org/manifesto

Reinartz, W., y Kumar, V. (2000). On the profitability of long-life customers in a noncontractual setting: An empirical investigation and implications for marketing. Journal of Marketing 2000, 64(4), pp. 17-35

SCImago, (2021). SJR - SCImago Journal \& Country Rank [Portal]. mayo 2020. De http:// www.scimagojr.com

Spinak, E. (1998). Indicadores cienciométricos. Ciência da Informação. 27. 141-148. 10.1590/ S0100-19651998000200006

Steinhoff, L., y Palmatier, R. (2021). Commentary: Opportunities and challenges of technology in relationship marketing. Australasian Marketing Journal, 2021, 29(2), pp. 111-117

Zhang, W.; Chintagunta, P., y Kalwani, M. (2021). Social Media, Influencers, and Adoption of an Eco-Friendly Product: Field Experiment Evidence from Rural China. Journal of Marketing, 2021, 85(3), pp. 10-27 